\title{
Role of weight functions in the study of a Dirichlet problem concerning the Laplace problem with parameter spectral and non-homogeneous in a cone
}

\author{
Hamrouni Ahmed ${ }^{1 *}$, Said Mohammed Saïd ${ }^{2}$ \\ ${ }^{1}$ First University, Echahid Hama Lakhder University, Eloued, Algeria \\ ${ }^{2}$ Second University, KasdiMerbah University, Ouargla, Algeria \\ *Corresponding author E-mail: hamrouni99@yahoo.fr
}

\begin{abstract}
In this work, we study the problem governed by the Laplacian operator perturbed by a spectral parameter and affected by a weight function in a cone. First, the study will be done in a space sector. We will show a theorem that links the study of this problem . This study is it provides original results in a richer functional space, more it covers the classical case.
\end{abstract}

Keywords: cone, Weight function, Laplacian operator.

\section{Introduction}

The aim of our work is to study the role played by the weight functions in

The study Elliptic problems of the form:

$\left\{\begin{array}{l}\Delta u+\lambda u=f \text { in } \mathbf{k}_{\varphi} \\ u=0 \text { on } \Gamma_{\varphi}\end{array}\right.$

Or $\mathbf{K}_{\phi}$ is a cone with boundary $\Gamma_{\phi}$, and $\mathrm{f}$ given in $\mathrm{L}^{2}\left(\mathbf{K}_{\phi}\right)$ and $\lambda \in \mathrm{R}$

$\Delta$ is the Laplace operator, it is defined by:

$\Delta u=\frac{\partial^{2} u}{\partial \rho^{2}}+\frac{1}{\rho} \frac{\partial u}{\partial \rho}+\frac{1}{\rho^{2}} \frac{\partial^{2} u}{\partial \theta^{2}}+\frac{\partial^{2} u}{\partial z^{2}}$

By rotation and by translation it is always possible to reduce the apex of $\mathbf{K}_{\phi}$ at the origin of the local coordinates. The solution of the problem:

$\left\{\begin{array}{l}\Delta u+\lambda u=f \text { in } \mathbf{K}_{\varphi} \\ u=0 \text { on } \Gamma_{\varphi}\end{array}\right.$

Is of the form:

$\mathrm{u}(\rho, \theta, \mathrm{z})=\mathrm{u}_{0}+\mathrm{a} \rho^{\frac{\mathrm{kn}}{\phi}} \sin \frac{\mathrm{k} \pi \theta}{\phi} \exp (\mathrm{kz}) \quad(\mathrm{a}$ cons tan $\mathrm{t})$.
This solution is not necessarily in $\mathrm{H}^{2}\left(\mathbf{K}_{\phi}\right)$ because $\frac{\partial^{2} \mathrm{u}}{\partial \rho^{2}} \notin$

$\mathrm{L}^{2}\left(\mathbf{K}_{\phi}\right)$ if $\phi>\pi$

But it is found that $\rho \frac{\partial^{2} \mathbf{u}}{\partial \rho^{2}} \in \mathrm{L}^{2}\left(\mathbf{K}_{\phi}\right) \forall \phi$, which gives us the idea of affecting the Laplacian of a weight function that is class $\mathrm{C}^{\infty}\left(\mathrm{K}_{\phi}\right)$ and does not null out in $\overline{\mathbf{K}}_{\phi}$.

\subsection{Some general results}

In [7] we find the solvability conditions of the Dirichlet problem in a cone $\mathbf{K}_{\phi} \subset \mathrm{R}^{\mathrm{n}}$ of vertex origin, relative to the problem:

$\left\{\begin{array}{l}\Delta u+\lambda u=f \text { in } \mathbf{K}_{\varphi} \\ u=0 \text { on } \Gamma_{\varphi}\end{array}\right.$

Where $\lambda$ is a complex parameter, called spectral parameter, belonging to $\mathrm{C}-\mathrm{R}_{+}$.

We construct a class of Sobolev spaces with weight for which the problem Dirichlet has a unique solution and is given the necessary conditions and sufficient to ensure the existence of this solution. The results will serve as "models" for more general problems.

The cace: $\lambda=0$

We generalize in a certain direction the works of [4] and [3] and [6].

The cace: $\lambda \neq 0$

It calls for a separate study of the case $\lambda=0$, the singularity at the end being different. The results generalize those of [3]. 
Remark 1.1: This weight function absorb the singularities which occur in the eighborhood of the top of $\mathbf{K}_{\phi}$, also it makes calculations easier. Considering the presence of the angular point on this top, to recover this, we multiply the operator $\Delta+\lambda$ by the function $\delta(\rho)$ which satisfies $\delta(\mathrm{x}, \mathrm{y}, \mathrm{z})=\rho^{\alpha}$ in the neighborhood of the origin.

\section{Position of the problem}

We propose to study the following problem:

$$
\left\{\begin{array}{l}
\rho^{\alpha}(\Delta u+\lambda u)=\rho^{\alpha} f \text { in } \mathbf{k}_{\phi} \\
u=0 \text { on } \Gamma_{\phi}
\end{array}\right.
$$

There fore the problem (3.1) becomes:

$$
\left\{\begin{array}{l}
\rho^{\alpha}\left(\frac{\partial^{2} u}{\partial \rho^{2}}+\frac{1}{\rho} \frac{\partial u}{\partial \rho}+\frac{1}{\rho^{2}} \frac{\partial^{2} u}{\partial \theta^{2}}+\frac{\partial^{2} u}{\partial z^{2}}+\lambda u\right)=\rho^{\alpha} f \text { in } \mathbf{K}_{\phi} \\
u=0 \text { on } \Gamma_{\phi}
\end{array}\right.
$$

We thus choose a functional space with weight that we note $\mathrm{E}_{\alpha}$ and defined by:

$$
\begin{aligned}
& \mathrm{E}_{\alpha}\left(\mathbf{K}_{\phi}\right) \\
& =\left\{\mathrm{u} \in \mathrm{H}^{1}\left(\mathbf{K}_{\phi}\right) \text { a Compact support and } \rho^{\alpha} \mathrm{D}^{2} \mathrm{u} \in \mathrm{L}^{2}\left(\mathrm{~K}_{\phi}\right)\right\}
\end{aligned}
$$

Where the symbol $\mathrm{D}^{2}$ denotes the partial derivatives of order 2 with respect to the variables $\rho$ and $\eta$ or $\frac{\partial}{\partial \eta}=\frac{1}{\rho} \frac{\partial}{\partial \theta}$. We will look for the solution of our problem, if it exists in the space $E_{\alpha}$. We shall see later that $\mathrm{E}_{\alpha}$ is an intermediate space between $\mathrm{H}^{1}$ and $\mathrm{H}^{2}$. We will provide the space $\mathrm{E}_{\alpha}\left(\mathbf{K}_{\phi}\right)$ of the following norm (called norm of the graph):

$$
\|\mathrm{u}\|_{\mathrm{E}_{\alpha}}=\|\mathrm{u}\|_{\mathrm{H}^{1}}+\sum_{|\beta|=2}\left\|\rho^{\alpha} \mathrm{D}^{\beta} \mathrm{u}\right\|
$$

We pose

$$
\mathrm{E}_{\alpha, 0}\left(\mathbf{K}_{\phi}\right)=\mathrm{E}_{\alpha}\left(\mathbf{K}_{\phi}\right) \cap \mathrm{H}_{0}^{1}\left(\mathbf{K}_{\phi}\right)
$$

\subsection{Property of space}

We showed in [2], the following results

$$
\begin{aligned}
& \mathrm{H}_{\mathrm{x}, \mathrm{y}, \mathrm{z}}^{2}\left(\mathbf{K}_{\phi}\right) \subset \mathrm{E}_{\alpha, 0}\left(\mathbf{K}_{\phi}\right) \subset \mathrm{H}_{\mathrm{x}, \mathrm{y}, \mathrm{z}}^{1}\left(\mathbf{K}_{\phi}\right) \\
& \mathrm{H}_{\mathrm{x}, \mathrm{y}, \mathrm{z}}^{1}\left(\mathbf{K}_{\phi}\right)=\mathrm{H}_{\rho, \eta, \mathrm{z}}^{1}\left(\mathbf{K}_{\phi}\right) \\
& \mathrm{H}_{\mathrm{x}, \mathrm{y}, \mathrm{z}}^{2}\left(\mathbf{K}_{\phi}\right) \subset \mathrm{H}_{\rho, \eta, \mathrm{z}}^{2}\left(\mathbf{K}_{\phi}\right)
\end{aligned}
$$

Proposition 2.1 If $u$ is in $E_{\alpha}\left(\mathbf{K}_{\phi}\right)$ so $\rho^{\alpha-1} \frac{\partial u}{\partial \rho}$ is in $L^{2}\left(\mathbf{K}_{\phi}\right)$.
Proof: We have if $\mathrm{u}$ is in $\mathrm{E}_{\alpha}\left(\mathbf{K}_{\phi}\right)$, so is compactly supported in $\overline{\mathbf{K}}_{\phi}$ and $\mathrm{u} \in \mathrm{H}^{1}\left(\mathbf{K}_{\phi}\right)$, and so $\frac{\partial \mathrm{u}}{\partial \rho}$ in $\mathrm{L}^{2}\left(\mathbf{K}_{\phi}\right)$ we pose $\mathrm{v}=\frac{\partial \mathrm{u}}{\partial \rho}$ and proof that $\rho^{\alpha-1}$ vis in $L^{2}\left(\mathbf{K}_{\phi}\right)$,so that:

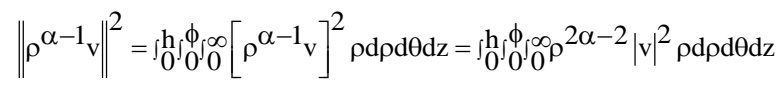

We have $\rho>1$ so $\|v\|^{2} \geq\left\|\rho^{\alpha-1} v\right\|^{2}$, because $\rho^{2 \alpha-2} \leq 1$ leads $(2 \alpha-2) \ln \rho \leq 0$ and like $0<\alpha<1$ we must necessarily have $\ln \rho \geq 0$ so $\rho \geq 1$.

So for $\rho \geq 1$, The proposition 2.1 is verified, and it remains the case $\rho<1$.

We have for $\rho<1$ :

$$
\begin{aligned}
& \int_{0}^{\mathrm{h}_{0}} \int_{0}^{\phi_{1}^{1}}\left[\rho^{\alpha-1} \mathrm{v}\right]^{2} \rho \mathrm{d} \rho \mathrm{d} \theta \mathrm{dz}= \\
& \int_{0}^{\mathrm{h}_{0}} \phi_{0}^{\phi_{j} \varepsilon_{0}} \rho^{2 \alpha-2}|\mathrm{v}|^{2} \rho \mathrm{d} \rho \mathrm{d} \theta \mathrm{dz}+\int_{0}^{\mathrm{h}_{0}} \phi_{0}^{\phi_{\rho}}{ }_{\varepsilon} \rho^{2 \alpha-2}|\mathrm{v}|^{2} \rho \mathrm{d} \rho \mathrm{d} \theta \mathrm{dz}
\end{aligned}
$$

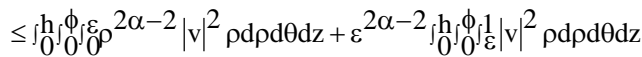

Because the function $\rho \rightarrow \rho^{2 \alpha-2}$ is decreasing and continuous on the compact $[\varepsilon, 1]$ so it is bounded and reaches its limits on this compact. It remains to study the integral $\int_{0}^{h_{0}} \rho_{0}^{\phi_{0}} \varepsilon_{0} \rho^{2 \alpha-2} v^{2} \rho d \rho d \theta d z$ for $\varepsilon$ sufficiently small and not null.

The function be analytic by serie $\rho \rightarrow \rho^{2 \alpha-2}=(1+\rho-1)^{2 \alpha-2}$. We have for $|\mathrm{X}|<1$ :

$(1+X)^{\beta}=\sum_{n=0}^{\infty} \frac{1}{n !} \beta(\beta-1)(\beta-2) \ldots(\beta-n+1) X^{n}$

And

$0<\rho<\varepsilon<1$ from Where $|\rho-1|<1$ we pose $X=\rho-1$ and $\beta=2 \alpha-2$ and apply development in serie entier, we will have:

$\int_{0}^{\mathrm{h}_{0}} \int_{0}^{\phi_{\delta}} \varepsilon_{0} \rho^{2 \alpha-2}|\mathrm{v}|^{2} \rho \mathrm{d} \rho \mathrm{d} \theta \mathrm{dz}=$

$\sum_{n=0}^{\infty} \frac{1}{n !}(2 \alpha-2)(2 \alpha-3) \ldots(2 \alpha-n-1)(\rho-1)^{n}|v|^{2} \rho d \rho d \theta d z$

From where:

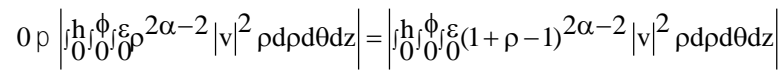

$\leq \sum_{\mathrm{n}=0}^{\infty} \frac{1}{\mathrm{n} !}(2 \alpha-2)(2 \alpha-3) \ldots(2 \alpha-\mathrm{n}-1) \int_{0}^{\mathrm{h}_{j}} \phi_{0}^{\phi}{ }_{0} \varepsilon|\mathrm{v}|^{2} \rho \mathrm{d} \rho \mathrm{d} \theta \mathrm{dz} \leq \mathrm{S}_{0}^{\mathrm{h}_{j}}{ }_{0}^{\phi_{j}}{ }_{0}|\mathrm{v}|^{2} \rho \mathrm{d} \rho \mathrm{d} \theta \mathrm{d} z$

Where $S$ is the sum of the series of general terms

$\frac{1}{n !}(2 \alpha-2)(2 \alpha-3) \ldots(2 \alpha-n-1)$. This series is surely convergent.

Then we have:

$0 \leq \int_{0}^{\mathrm{h}_{j}} \phi_{0} \phi_{0} \rho^{2 \alpha-2}|\mathrm{v}|^{2} \rho \mathrm{d} \rho \mathrm{d} \theta \mathrm{dz} \leq \mathrm{S}_{0}^{\mathrm{h}_{j}} \phi_{0}^{\phi_{\delta}} \varepsilon|\mathrm{v}|^{2} \rho \mathrm{d} \rho \mathrm{d} \theta \mathrm{dz}$

And since $\mathrm{v} \in \mathrm{L}^{2}$ we have the desired result.

Proposition2.2. There exists a constant $\mathrm{C}>0, \mathrm{C}$ depends on the open $\mathbf{K}_{\phi}$ such that: 
$\left\|\rho^{\alpha-1} \frac{\partial u}{\partial \rho}\right\|^{2} \leq C\left\|\rho^{\alpha-1} \frac{\partial^{2} u}{\partial \rho \partial \theta}\right\|^{2}$

Proof: From proposition2.1we have $\mathrm{u} \in \mathrm{L}^{2}\left(\mathrm{~K}_{\phi}\right)$ so that $\rho^{\alpha-1} \frac{\partial u}{\partial \rho} \in \mathrm{L}^{2}\left(\mathbf{K}_{\phi}\right)$ we posons $\mathrm{v}=\rho^{\alpha-1} \frac{\partial \mathrm{u}}{\partial \rho}$ the functions integrable with respect to the measure and being compactly supported in $\overline{\mathrm{K}_{\phi}}$, the function $\mathrm{v}$ is also. It's about of a proof The following inequality:

$\|\mathrm{v}\| \leq \mathrm{C}\left\|\frac{\partial \mathrm{v}}{\partial \theta}\right\|$

$\mathrm{v}(\mathrm{x}, \mathrm{y}, \mathrm{z})=\int_{0}^{\theta} \frac{\partial \mathrm{v}}{\partial \theta}(\rho \cos \mathrm{t}, \rho \sin \theta, \mathrm{z}) \mathrm{dt}$

$|\mathrm{v}(\mathrm{x}, \mathrm{y}, \mathrm{z})|^{2} \leq \mathrm{C} \int_{0}^{\theta} \frac{\partial \mathrm{v}}{\partial \theta}|(\rho \cos \mathrm{t}, \rho \sin \theta, \mathrm{z})|^{2} \mathrm{dt}$

So that:

$\int_{0}^{\mathrm{h}_{j}}{ }_{0}^{\phi_{j}} \rho_{0}^{\infty}|\mathrm{v}(\mathrm{x}, \mathrm{y}, \mathrm{z})|^{2} \rho \mathrm{d} \rho \mathrm{d} \theta \mathrm{dz} \leq \int_{0}^{\theta} \int_{0}^{\mathrm{h}_{j}} \phi_{0}^{\phi_{0}}{ }_{0}^{\infty} \frac{\partial \mathrm{v}}{\partial \theta}|(\rho \cos \mathrm{t}, \rho \sin \theta, \mathrm{z})|^{2} \rho \mathrm{d} \rho \mathrm{d} \theta \mathrm{dzdt}$

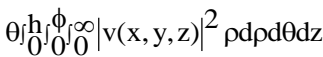

So:

$$
\left\|\rho^{\alpha-1} \frac{\partial u}{\partial \rho}\right\|^{2} \leq C\left\|\rho^{\alpha-1} \frac{\partial^{2} u}{\partial \rho \partial \theta}\right\|
$$

The result is verified.

Proposition2.3: The space $\mathrm{E}_{\alpha, 0}\left(\mathbf{K}_{\phi}\right)$ Can be written in the following form:

$\mathrm{E}_{\alpha, 0}\left(\mathbf{K}_{\phi}\right)=\left\{\mathrm{u} \in \mathrm{H}_{0}^{1}\left(\mathbf{K}_{\phi}\right)\right.$ such as $\left.\rho^{\alpha} \mathrm{u} \in \mathrm{H}_{0}^{2}\left(\mathbf{K}_{\phi}\right)\right\}$

Where the functions are compactly supported in $\overline{\mathbf{K}}_{\phi}$.

Proof

Let

$\mathrm{G}_{\alpha, 0}\left(\mathbf{k}_{\phi}\right)=\left\{\mathrm{u} \in \mathrm{H}_{0}^{1}\left(\mathbf{k}_{\phi}\right)\right.$ such as $\left.\rho^{\alpha} \mathrm{u} \in \mathrm{H}_{0}^{2}\left(\mathbf{K}_{\phi}\right)\right\}$

And made proof by double inclusion $\mathrm{G}_{\alpha, 0}\left(\mathbf{k}_{\phi}\right) \subset \mathrm{E}_{\alpha, 0}\left(\mathbf{K}_{\phi}\right)$ and $\mathrm{G}_{\alpha, 0}\left(\mathbf{K}_{\phi}\right) \supset \mathrm{E}_{\alpha, 0}\left(\mathbf{K}_{\phi}\right)$ that these two spaces coincide .L and $\mathrm{u} \in \mathrm{G}_{\alpha, 0}\left(\mathbf{k}_{\phi}\right)$ so that $\mathrm{u} \in \mathrm{H}_{0}^{1}\left(\mathbf{k}_{\phi}\right)$ and $\rho^{\alpha} \mathrm{u} \in \mathrm{H}_{0}^{2}\left(\mathbf{K}_{\phi}\right)$ from where: $\rho^{\alpha} \frac{\partial u}{\partial \rho}+\alpha \rho^{\alpha-1} u \in H_{0}^{1}\left(\mathbf{K}_{\phi}\right)$ and so $\rho^{\alpha} \frac{\partial^{2} u}{\partial \rho^{2}}+2 \alpha \rho^{\alpha-1} u \in L^{2}\left(\mathbf{K}_{\phi}\right)$ and as theproposition $2.1 \rho^{\alpha-1} \frac{\partial u}{\partial \rho} \in \mathrm{L}^{2}\left(\mathbf{K}_{\phi}\right)$ so that $\rho^{\alpha-1} \frac{\partial^{2} \mathrm{u}}{\partial \rho^{2}} \in \mathrm{L}^{2}\left(\mathbf{K}_{\phi}\right)$

Similarly, $\rho^{\alpha} \mathrm{u} \in \mathrm{H}_{0}^{2}\left(\mathbf{K}_{\phi}\right.$ implies that $\rho^{\alpha} \frac{1}{\rho} \frac{\partial \mathrm{u}}{\partial \theta} \in \mathrm{H}_{0}^{1}\left(\mathbf{K}_{\phi}\right)$, so that $\rho^{\alpha-2} \frac{\partial^{2} \mathbf{u}}{\partial \theta^{2}} \in \mathrm{L}^{2}\left(\mathbf{K}_{\phi}\right)$. We also have: $\rho^{\alpha} \mathrm{u} \in \mathrm{H}_{0}^{2}\left(\mathbf{K}_{\phi}\right)$ implies that $\frac{1}{\rho} \frac{\partial^{2}}{\partial \rho \partial \theta}\left(\rho^{\alpha} \mathbf{u}\right) \mathrm{L}^{2}\left(\mathbf{K}_{\phi}\right)$

And like:

$\rho^{\alpha} \frac{1}{\rho} \frac{\partial \mathrm{u}}{\partial \theta} \in \mathrm{H}_{0}^{1}\left(\mathbf{K}_{\phi}\right) \subset \mathrm{L}^{2}\left(\mathbf{K}_{\phi}\right)$ So $\rho^{\alpha-1} \frac{\partial^{2} \mathrm{u}}{\partial \rho \partial \theta} \in \mathrm{L}^{2}\left(\mathbf{K}_{\phi}\right)$

We have the result $\mathrm{G}_{\alpha, 0}\left(\mathrm{~K}_{\phi}\right) \subset \mathrm{E}_{\alpha, 0}\left(\mathrm{~K}_{\phi}\right)$.

And by the same process one shows the inclusion in the other direction.

Lemma2.4: For all function $\mathrm{u}$ in $\mathrm{E}_{\alpha, 0}\left(\mathbf{K}_{\phi}\right)$, We have the following inequality:

$(2 \alpha-1)\left\|\rho^{\alpha-1} \frac{\partial \mathrm{u}}{\partial \rho}\right\| \leq \rho^{\alpha} \frac{\partial^{2} \mathrm{u}}{\partial \rho^{2}} \|$

We have $\left\|\rho^{\alpha-1} \frac{\partial u}{\partial \rho}+\rho^{\alpha} \frac{\partial^{2} u^{2}}{\partial \rho^{2}}\right\| \geq 0$, which means that:

$\left\|\rho^{\alpha-1} \frac{\partial u}{\partial \rho}\right\|+\left\|\rho^{\alpha} \frac{\partial^{2} u}{\partial \rho^{2}}\right\|+2 \int_{K_{b}} \rho^{2 \alpha} \frac{\partial u}{\partial \rho} \frac{\partial^{2} u}{\partial \rho^{2}} d \rho d \theta d z \geq 0$

And we have:

$2 \int_{\mathbf{K}} \rho^{2 \alpha} \frac{\partial u}{\partial \rho} \frac{\partial^{2} u}{\partial \rho^{2}} d \rho d \theta d z=2 \int \rho_{K_{b}} \rho^{2 \alpha} \frac{\partial u}{\partial \rho}\left(\frac{\partial u}{\partial \rho}\right)^{2} \operatorname{d} \rho d \theta d z$.

We integrate this last integral by parts, and taking into account that the functions are with compact support in $\overline{\mathrm{K}_{\phi}}$, so that:

$2 \int_{\mathrm{K}_{i}} \rho^{2 \alpha} \frac{\partial \mathrm{u}}{\partial \rho} \frac{\partial^{2} \mathrm{u}}{\partial \rho^{2}} \mathrm{~d} \rho \mathrm{d} \theta \mathrm{d} z=-2\left\|\rho^{\alpha-1} \frac{\partial \mathrm{u}}{\partial \rho}\right\|^{2}$

Hence the expression

$\left\|\rho^{\alpha-1} \frac{\partial u}{\partial \rho}+\rho^{\alpha} \frac{\partial^{2} u^{2}}{\partial \rho^{2}}\right\|^{2} \geq 0$

So:

$(2 \alpha-1)\left\|\rho^{\alpha-1} \frac{\partial u}{\partial \rho}\right\| \leq \rho^{\alpha} \frac{\partial^{2} u}{\partial \rho^{2}} \|$

\section{Study of the existence of the solution}

We propose to study the solution of the problem (2.1) in plane sector. $\mathbf{K}_{\phi}$. This study will be extended to the cone by using a partition of the unity of this one.

Theorem3.1:If $\mathrm{f}$ is a function given in $\mathrm{L}^{2}\left(\mathrm{~K}_{\phi}\right)$ the problem (2.1) admits an unique solution in space $\mathrm{E}_{\alpha}\left(\mathbf{K}_{\phi}\right)$ if and only if the following problem:

$\left\{\begin{array}{l}\rho^{\alpha} \Delta \mathrm{u}=\rho^{\alpha} \mathrm{f}=\mathrm{g} \quad \text { in } \mathbf{K}_{\phi} \\ \mathrm{u}=0 \quad \text { on } \Gamma_{\phi}\end{array}\right.$

Admits an unique solution in space $\mathrm{E}_{\alpha}\left(\mathbf{K}_{\phi}\right)$ 
Proof: It is evident that if the Problem(2.1) admits an unique solution in space $\mathrm{E}_{\alpha}\left(\mathbf{K}_{\phi}\right)$ then the Problem (3.1) admits an unique solution in space $\mathrm{E}_{\alpha}\left(\mathrm{K}_{\phi}\right)$,because the problem (3.1) is a particular case of the problem (2.1), it suffices to pose $\lambda=0$ in (2.1). We will prove the reciprocal. We propose to seek the solution if it exists for the problem (3.1) in the space $\mathrm{E}_{\alpha}\left(\mathrm{K}_{\phi}\right)$ to study the existence of the solution of this problem, we will study the image of the operator $A=\rho^{\alpha} \Delta$ in space $L^{2}\left(K_{\phi}\right)$. To establish that the image of $A$ is closed in $\mathrm{L}^{2}\left(\mathbf{K}_{\phi}\right)$, we will be the following inequality : There is a constant $\mathrm{C}>0$ such that:

$$
\|\mathrm{u}\|_{\mathrm{E}_{\alpha, 0}\left(\mathbf{K}_{\phi}\right)} \leq \mathrm{C}\left\{\|\mathrm{u}\|_{\mathrm{L}^{2}\left(\mathbf{K}_{\phi}\right)}\left(\mathbf{K}_{\phi}\right)+\|\mathrm{u}\|_{\mathrm{H}_{0}^{1}\left(\mathbf{K}_{\phi}\right)}\right\}
$$

The inequality (3.2), is deduced from the following lemma showed in [5]

Lemma3.2: of Peetre:: If $\mathrm{X}, \mathrm{Y}, \mathrm{Z}$ are three Banach reflexivexive spaces such that $\mathrm{X} \subset \mathrm{Y}$ with compact injection, and $\mathrm{P}$ is a continuous linear operator from $\mathrm{X}$ to $\mathrm{Z}$, then the following conditions are equivalent:

1) the image of $P$ is closed in $Z$ and kernel of $P$ has finite dimension

2) there is constant $\mathrm{C}>0$ such that:

$\|\mathrm{u}\|_{\mathrm{X}} \leq \mathrm{C}\left\{\|\mathrm{u}\|_{\mathrm{Z}}\left(\mathrm{k}_{\phi}\right)+\|\mathrm{u}\|_{\mathrm{Y}}\right\}$

For our problem we consider $\mathrm{P}=\mathrm{A} ; \mathrm{X}=\mathrm{E}_{\alpha, 0}\left(\mathrm{~K}_{\phi}\right) ; \mathrm{Y}=\mathrm{H}_{0}^{1}\left(\mathbf{K}_{\phi}\right)$ and $\mathrm{Z}=\mathrm{L}^{2}\left(\mathrm{~K}_{\phi}\right)$, that all the hypothesis of the Lemma 4.1 are verified, so to obtain (1) in the Lemma 4.1, we will prove (2), then we will establish the inequality (3.2).We prove now the inequality (3.2).For this we be calculate $\left\|\rho^{\alpha} \Delta u\right\|^{2}$.

$\left\|\rho^{\alpha} \Delta u\right\|^{2}=\left\|\rho^{\alpha} \frac{\partial^{2} u}{\partial \rho^{2}}\right\|^{2}+\left\|\rho^{\alpha-1} \frac{\partial u}{\partial \rho}\right\|^{2}$

$+\left\|\rho^{\alpha-2} \frac{\partial^{2} u}{\partial \theta^{2}}\right\|^{2}+\left\|\rho^{\alpha} \frac{\partial^{2} u}{\partial z^{2}}\right\|^{2}+2(I+J+K+L+M+N)$

Or

$\mathrm{I}=\int_{\mathbf{K}_{\phi}} \rho^{2 \alpha} \frac{\partial \mathrm{u}}{\partial \rho} \frac{\partial^{2} \mathrm{u}}{\partial \rho^{2}} \mathrm{~d} \rho \mathrm{d} \theta \mathrm{dz}$

$J=\int_{\mathbf{K}_{\phi}} \rho^{2 \alpha-1} \frac{\partial^{2} \mathbf{u}}{\partial \rho^{2}} \frac{\partial^{2} \mathbf{u}}{\partial \theta^{2}} \mathrm{~d} \rho \mathrm{d} \theta \mathrm{dz} ;$

$\mathrm{K}=\int_{\mathbf{K}_{\phi}} \rho^{2 \alpha-2} \frac{\partial u}{\partial \rho} \frac{\partial^{2} u}{\partial \theta^{2}} \mathrm{~d} \rho \mathrm{d} \theta \mathrm{d} z$

$\mathrm{L}=\int_{\mathbf{K}_{\phi}} \rho^{2 \alpha+1} \frac{\partial^{2} \mathbf{u}}{\partial \rho^{2}} \frac{\partial^{2} \mathbf{u}}{\partial \mathbf{z}^{2}} \mathrm{~d} \rho \mathrm{d} \theta \mathrm{d} \mathbf{z}$;

$\mathbf{M}=\int_{\mathbf{K}_{\phi}} \rho^{2 \alpha} \frac{\partial u}{\partial \rho} \frac{\partial^{2} \mathbf{u}}{\partial \mathbf{z}^{2}} \mathrm{~d} \rho \mathrm{d} \theta \mathrm{dz}$;

$\mathrm{N}=\int_{\mathbf{K}_{\phi}} \rho^{2 \alpha-1} \frac{\partial^{2} \mathbf{u}}{\partial \theta^{2}} \frac{\partial^{2} \mathbf{u}}{\partial \mathbf{z}^{2}} \mathrm{~d} \rho \mathrm{d} \theta \mathrm{dz}$
We calculate each of the expressions I, J , K, L , M , N by integration by parties and taking into account boundary conditions and the fact that functions are compactly supported we get:

$I=-\alpha\left\|\rho^{\alpha-1} \frac{\partial u}{\partial \rho}\right\|^{2}$

And

$\mathrm{J}+\mathrm{K}=(2-2 \alpha)^{2}\left\|\rho^{\alpha-1} \frac{1}{\rho} \frac{\partial \mathrm{u}}{\partial \theta}\right\|^{2}+\left\|\rho^{\alpha-1} \frac{\partial^{2} \mathrm{u}}{\partial \rho \partial \theta}\right\|^{2}$

And

$\mathrm{L}+\mathrm{M}=\left\|\rho^{\alpha} \frac{\partial^{2} \mathrm{u}}{\partial \rho \partial \mathrm{z}}\right\|^{2}-2 \alpha(2 \alpha+1)\left\|\rho^{\alpha} \frac{\partial \mathrm{u}}{\partial \mathrm{z}}\right\|^{2} ; \mathrm{N}=-\alpha\left\|\rho^{\alpha} \frac{\partial^{2} \mathrm{u}}{\partial \theta \partial \mathrm{z}}\right\|^{2}$

So:

$\left\|\rho^{\alpha} \Delta u\right\|^{2}=\left\|\rho^{\alpha} \frac{\partial^{2} \mathrm{u}}{\partial \rho^{2}}\right\|^{2}+\left\|\rho^{\alpha-1} \frac{\partial u}{\partial \rho}\right\|^{2}+\left\|\rho^{\alpha-2} \frac{\partial^{2} u}{\partial \theta^{2}}\right\|^{2}+\left\|\rho^{\alpha} \frac{\partial^{2} u}{\partial z^{2}}\right\|^{2}$

$+2\left[-\alpha\left\|\rho^{\alpha-1} \frac{\partial u}{\partial \rho}\right\|^{2}+(2-2 \alpha)^{2}\left\|\rho^{\alpha-1} \frac{1}{\rho} \frac{\partial u}{\partial \theta}\right\|^{2}+\left\|\rho^{\alpha-1} \frac{\partial^{2} u}{\partial \rho \partial \theta}\right\|^{2}\right.$

$\left.+\left\|\rho^{\alpha} \frac{\partial^{2} u}{\partial \rho \partial z}\right\|^{2}-2 \alpha(2 \alpha+1)\left\|\rho^{\alpha} \frac{\partial u}{\partial z}\right\|^{2}+N=-\alpha\left\|\rho^{\alpha} \frac{\partial^{2} u}{\partial \theta \partial z}\right\|^{2}\right]$

So we have:

$\left\|\rho^{\alpha} \Delta \mathrm{u}\right\|^{2}+\|\mathrm{u}\|_{\mathrm{H}^{1}}^{2}=\|\mathrm{u}\|_{\mathrm{E}_{\alpha, 0}}{ }^{2}+$

$(1-2 \alpha)\left\|\rho^{\alpha-1} \frac{\partial u}{\partial \rho}\right\|^{2}+2(2-2 \alpha)^{2}\left\|\rho^{\alpha-1} \frac{\partial u}{\partial \rho}\right\|^{2}+$

$2\left\|\rho^{\alpha-1} \frac{\partial^{2} u}{\partial \rho \partial \theta}\right\|^{2}+2\left\|\rho^{\alpha} \frac{\partial^{2} u}{\partial \rho \partial z}\right\|^{2}-$

$4 \alpha(2 \alpha+1)\left\|\rho^{\alpha} \frac{\partial u}{\partial z}\right\|^{2}+2\left\|\rho^{\alpha} \frac{\partial^{2} u}{\partial \theta \partial z}\right\|^{2}$

$\geq\|\mathrm{u}\|_{\mathrm{E}_{\alpha, 0}}^{2}+(1-2 \alpha)\left\|\rho^{\alpha-1} \frac{\partial \mathrm{u}}{\partial \rho}\right\|^{2}$

$+2\left\|\rho^{\alpha-1} \frac{\partial^{2} u}{\partial \rho \partial \theta}\right\|^{2}+2\left\|\rho^{\alpha} \frac{\partial^{2} u}{\partial \rho \partial z}\right\|^{2}$

$-4 \alpha(2 \alpha+1)\left\|\rho^{\alpha} \frac{\partial u}{\partial z}\right\|^{2}$

Because:

$2(2-2 \alpha)^{2}\left\|\rho^{\alpha-1} \frac{\partial u}{\partial \rho}\right\|^{2}+2\left\|\rho^{\alpha} \frac{\partial^{2} u}{\partial \theta \partial z}\right\|^{2} \geq 0$ 
And according to propositions 2.2 and lemma 2.4 we have the desired result by choosing a suitable constant $\mathrm{C}_{\phi}$. We thus have a constant for the case $\alpha \geq \frac{1}{2}$ and another constant for the case $\alpha<\frac{1}{2}$.

$$
\mathrm{C}_{\phi}\left\{\|\mathrm{u}\|_{\mathrm{L}^{2}\left(\mathbf{K}_{\phi}\right)}\left(\mathbf{K}_{\phi}\right)+\|\mathrm{u}\|_{\mathrm{H}_{0}^{1}\left(\mathbf{K}_{\phi}\right)}\right\} \geq\|\mathrm{u}\|_{\mathrm{E}_{\alpha, 0}\left(\mathbf{K}_{\phi}\right)} .
$$

There is existe therefore a constant $\mathrm{C}_{\alpha, \phi}>0$, which depends on $\alpha$ and $\phi$, which checks the inequality (3.4).Thus according to inequality(3.4) and the lemma 3.2, we deduce that the problem (3.1) admit a solution in $\mathrm{E}_{\alpha, 0}\left(\mathrm{~K}_{\phi}\right)$. Now we prove that the inequality (3.4) can be extended to the problem (2.1).

We have:

$$
\begin{aligned}
& \left\|\rho^{\alpha} \Delta u\right\|_{L^{2}\left(K_{\phi}\right)} \\
& =\left\|\rho^{\alpha} \Delta u+\rho^{\alpha} \lambda u-\rho^{\alpha} \lambda u\right\|_{L^{2}\left(K_{\phi}\right)} \leq\|A u\|_{L^{2}\left(K_{\phi}\right)}+\left\|\rho^{\alpha} \lambda u\right\|_{L^{2}\left(K_{\phi}\right)}
\end{aligned}
$$

Which implies

$$
\left\|\rho^{\alpha} \Delta \mathrm{u}\right\|_{\mathrm{L}^{2}\left(K_{\phi}\right)}^{2} \leq\left(\|A \mathrm{u}\|_{\mathrm{L}^{2}\left(\mathrm{~K}_{\phi}\right)}+\left\|\rho^{\alpha} \lambda \mathrm{u}\right\|_{\mathrm{L}^{2}\left(\mathrm{~K}_{\phi}\right)}\right)^{2}
$$

So that:

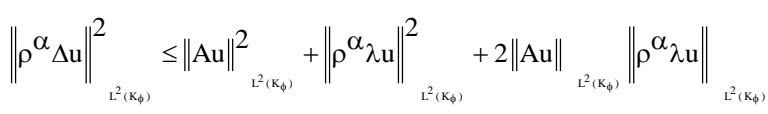

According to the elementary formula:

$$
(a-b)^{2}=a^{2}+b^{2}-2 a b \text { So } a^{2}+b^{2} \geq 2 a b
$$

We take $a=\|A u\|_{L^{2}\left(\mathbf{K}_{\phi}\right)}$ and $b=\left\|\rho^{\alpha} \lambda u\right\|_{L^{2}\left(\mathbf{K}_{\phi}\right)}$ which give:

$$
\left\|\rho^{\alpha} \Delta \mathrm{u}\right\|_{\mathrm{L}^{2}\left(\mathrm{~K}_{\phi}\right)}^{2} \leq 2\left(\|\mathrm{Au}\|_{\mathrm{L}^{2}\left(\mathrm{~K}_{\phi}\right)}^{2}+\left\|\rho^{\alpha} \lambda \mathrm{u}\right\|_{\mathrm{L}^{2}\left(\mathrm{~K}_{\phi}\right)}^{2}\right)
$$

So that

$$
\left\|\rho^{\alpha} \Delta \mathrm{u}\right\|_{\mathrm{L}^{2}\left(\mathrm{~K}_{\phi}\right)}^{2}+\|\mathrm{u}\|_{\mathrm{H}_{0}^{1}}^{2} \leq 2\left(\|\mathrm{Au}\|_{\mathrm{L}^{2}\left(\mathrm{~K}_{\phi}\right)}^{2}+\left\|\rho^{\alpha} \lambda \mathrm{u}\right\|_{\mathrm{L}^{2}\left(\mathrm{~K}_{\phi}\right)}^{2}\right)+\|\mathrm{u}\|_{\mathrm{H}_{0}^{1}}^{2}
$$

So

$$
\left\|\rho^{\alpha} \Delta \mathrm{u}\right\|_{\mathrm{L}^{2}\left(\mathrm{~K}_{\varphi}\right)}^{2}+\|\mathrm{u}\|_{\mathrm{H}_{0}^{\prime}} \leq 2\left(\|\mathrm{Au}\|_{\mathrm{L}^{2}\left(\mathrm{~K}_{Q}\right)}^{2}+\left\|\rho^{\alpha} \lambda \mathrm{u}\right\|_{\mathrm{L}^{2}\left(\mathrm{~K}_{\varphi}\right)}^{2}\right)+\left\|\rho^{\alpha} \lambda \mathrm{u}\right\|_{\mathrm{H}_{\mathrm{o}}^{\prime}}^{2}
$$

We have the function has compact support in $\overline{\mathbf{K}}_{\phi}$ Then there existe $\rho_{0}>0 \rho_{0}$ finite as:

$\left\|\rho^{\alpha} \lambda \mathrm{u}\right\|_{\mathrm{L}^{2}\left(K_{\phi}\right)}^{2} \leq|\lambda|^{2} \rho_{0}{ }^{2 \alpha}\|\mathrm{u}\|_{\mathrm{H}_{0}^{1}}$

And injection of $\mathrm{H}_{0}^{1}$ in $\mathrm{L}^{2}$ is continuous[4] , therefore the above inequality:

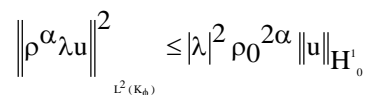

So that (3.5):

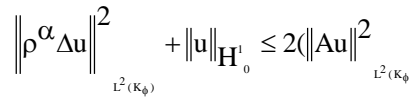

$$
\begin{aligned}
& \left.+\left\|\rho^{\alpha} \lambda \mathrm{u}\right\|_{\mathrm{L}^{2}\left(\mathrm{~K}_{\phi}\right)}^{2}\right)+|\lambda|^{2} \rho_{0}{ }^{2 \alpha}\|\mathrm{u}\|_{\mathrm{H}^{1}}
\end{aligned}
$$

From the inequalities (3.4) and (3.6) we deduce:

$\frac{1}{\mathrm{C}}\|\mathrm{u}\|_{\mathrm{E}_{\alpha, 0}} \leq 2\left(\|\mathrm{Au}\|_{\mathrm{L}^{2}\left(\mathrm{~K}_{\phi}\right)}^{2}+\left\|\rho^{\alpha} \lambda \mathrm{u}\right\|_{\mathrm{L}^{2}\left(\mathrm{~K}_{\phi}\right)}^{2}\right)+|\lambda|^{2} \rho_{0} 2 \alpha\|\mathrm{u}\|_{\mathrm{H}^{1}}$

So:

$\frac{1}{\mathrm{C}}\|\mathrm{u}\|_{\mathrm{E}_{\alpha, 0}} \leq 2\|\mathrm{Au}\|_{\mathrm{L}^{2}\left(\mathrm{~K}_{\phi}\right)}^{2}+\left(2+2|\lambda|^{2} \rho_{0}{ }^{2 \alpha}\right)\|\mathrm{u}\|_{\mathrm{H}_{o}^{\prime}}$

Where $\lambda$ Is any non null constant. If one takes a constant $\mathrm{C}_{1}=\max \left(2,2+2|\lambda|^{2} \rho_{0}^{2 \alpha}\right)$ we have: There exists a constant $\mathrm{K}=\mathrm{CC}_{1}>0$ such that:

$\|\mathrm{u}\|_{\mathrm{E}_{\alpha, 0}} \leq \mathrm{K}\left\{\|\mathrm{Au}\|_{\mathrm{L}^{2}\left(\mathrm{~K}_{\phi}\right)}^{2}+\|\mathrm{u}\|_{\mathrm{H}_{0}^{\prime}}\right\}$

Thus according to the inequality (3.8) and the Lemma 3.2, we deduce that the image of the operator $\mathrm{A}$ is closed in $\mathrm{L}^{2}\left(\mathrm{~K}_{\phi}\right)$ and the kernel of the problem (2.1) has a finite dimension, thereafter the problem (2.1) admits at least a solution in $\mathrm{E}_{\alpha, 0}\left(\mathbf{K}_{\phi}\right)$

\section{Study of the uniqueness of the solution}

We propose to study the uniqueness of the solution of problem (3.2). For this we calculate its kernel in space $\mathrm{E}_{\alpha, 0}\left(\mathbf{K}_{\phi}\right)$ the elements of the kernel of the problem (3.2) are solution of the following problem:

$\left\{\begin{array}{l}\rho^{\alpha}(\Delta u+\lambda u)=0 \quad \text { in } \mathbf{k}_{\phi} \\ u=0 \quad \text { on } \Gamma_{\phi}\end{array}\right.$

According to $\rho^{\alpha} \neq 0$ in $\mathbf{K}_{\phi}$, then the problem (4.1) can be written as:

$\left\{\begin{array}{l}\Delta \mathrm{u}+\lambda \mathrm{u}=0 \text { in } \mathbf{k}_{\phi} \\ \mathrm{u}=0 \quad \text { on } \Gamma_{\phi}\end{array}\right.$

We will study the uniqueness of the solution of the problem (3.1), we have:

$$
\Delta \mathrm{u}=0 \Rightarrow\langle\Delta \mathrm{u}, \mathrm{u}\rangle=-\left\|\frac{\partial \mathrm{u}}{\partial \mathrm{x}}\right\|^{2}-\left\|\frac{\partial \mathrm{u}}{\partial \mathrm{y}}\right\|^{2}-\left\|\frac{\partial \mathrm{u}}{\partial \mathrm{z}}\right\|^{2}=0
$$

Then $\mathrm{u}$ is a constant in $\mathrm{H}^{1}\left(\mathrm{~K}_{\phi}\right)$, according to $\mathrm{u}=0$ on $\Gamma_{\phi}$ we deduce that $\mathrm{u}=0$ in $\mathrm{H}^{1}\left(\mathbf{K}_{\phi}\right)$ then $\mathrm{u}=0$ in $\mathrm{E}_{\boldsymbol{\alpha}, 0}\left(\mathbf{K}_{\phi}\right)$.

Now we study the uniqueness of the solution of the problem (2.1), for this we calculate its kernel, we remark that: 
$\mathrm{Au}=0 \Rightarrow\langle\Delta \mathrm{u}+\lambda \mathrm{u}, \mathrm{u}\rangle=-\left\|\frac{\partial \mathrm{u}}{\partial \mathrm{x}}\right\|^{2}-\left\|\frac{\partial \mathrm{u}}{\partial \mathrm{y}}\right\|^{2}-\left\|\frac{\partial \mathrm{u}}{\partial \mathrm{z}}\right\|^{2}+\lambda\|\mathrm{u}\|^{2}=0$

We distinguish two cases:

Case 1: $\lambda<0$ Therefore $\mathrm{u}=0$ in $\mathrm{H}^{1}\left(\mathbf{K}_{\phi}\right)$ then $\mathrm{u}=0$ in $\mathrm{E}_{\alpha, 0}\left(\mathbf{K}_{\phi}\right)$

Case 2: $\lambda>0$ we pose $\beta^{2}=\lambda$ Then $\|\nabla \mathrm{u}\|_{\mathrm{L}^{2}\left(\mathbf{K}_{\phi}\right)}^{2}=\beta^{2}\|\mathrm{u}\|_{\mathrm{L}^{2}\left(\mathbf{K}_{\phi}\right)}^{2}$ using the fact that $\|\nabla \mathrm{u}\|_{\mathrm{L}^{2}\left(\mathrm{~K}_{\phi}\right)}^{2}$ is a norm equivalent to the usual norm of $\mathrm{H}^{1}\left(\mathbf{K}_{\phi}\right)$ we deduce that $\mathrm{u}=0$ in $\mathrm{H}^{1}\left(\mathbf{K}_{\phi}\right)$ then $\mathrm{u}=0$ in $\mathrm{E}_{\alpha, 0}\left(\mathbf{K}_{\phi}\right) \mathrm{We}$ deduce that the problem (2.1) admits an unique solution in $\mathrm{E}_{\alpha, 0}\left(\mathbf{K}_{\phi}\right)$.

This completes the proof of the theorem 3.1

\section{References}

[1] A. Avantaggiati Et M. Troisi, spazidisobolev con peso e problemielliptici in angolo, dimat .pura e appl , 1973.

[2] M. Borsuk Et V. Kondratiev, Elliptic Boundary Value Problems of Second Order in Piecewise Smooth Domains Elsevier B, V North-Holland, 2006.

[3] P. Grisvard, Problème de Dirichlet dans un cône .Ricerche di Matematica, vol. XX, p. 175-192, 1971.

[4] V. Khoan, Distributions analyse de Fourier operateur aux dérivéespartielles, Vuibert, 1972 .

[5] J. L. LIONS and E. MAGENES, Problems in Extreme Cases Nonhomogeneous and Applications, Vol. 1, Dunod, 1968

[6] V. A. Kondratiev, Boundary value problems for elliptic equations Math, soc 1967.

[7] Pham The Lai, Problème de Dirichlet dans un cône avec paramètre spectral pour uneclassed'espace de Sobolev a poids, SEDP exp . N_6 p.1-14, 1979. 